\title{
Internet of Things (IOT): Architectures, Protocols and Frameworks, Applications and Challenges in Healthcare
}

\author{
Nisrine MACHRAFI, Jalal LAASSIRI
}

\begin{abstract}
The world of internet of things is a world where all devices are connected to internet by different technologies and protocols, in different domain of application. The world of internet of thing creates an interaction between object (physical things) and internet (digital technologies), using actuators or sensors or others, and collaborates with each other. The connected device can be used as a collecting object for data and it's the input case of IoT or as an analyzing device and it's the output case of the IoT. More than that there is different domain of application of internet of things, different protocols and frameworks used and the most important is that there are different architecture design depending of the use of the connected device. We will present a comparison between architectures, protocols (characteristic, execution time and performance), we discuss the frameworks and their challenges and limits. Finally, we will give synopsis evolution of IoT in Healthcare.
\end{abstract}

Keywords: Internet of Things, Architectures, Protocols, frameworks, application.

\section{INTRODUCTION}

The Internet of things had got an important role in our daily activities, covering a lot of field as homes, automobile, agriculture, and healthcare and more activities get easer from user's perspective. But as a developer and system design, as a scientific we know how much challenges IoT face, like how much this object are secure to use, which level of security are provided to users.

The challenge of developers in front of all existing IoT architecture, and more even with chosen architecture, every layer can use different protocol, and finally use different frameworks. The complexity of this challenges had led helps the Internet of Things word to a quick evolution of protocols and frameworks which led to more challenging choices or more creativity. And recently, different frameworks have been «launched" in the Iot different field by the research community to make their development easer and to support it.

\section{A. History and major events of IoT}

The United states Defense Advanced Research Projects (DARPA) has developed ARPANET which is the precursor

Revised Manuscript Received on March 22, 2019.

* Correspondence Author

Nisrine MACHRAFI*, Department of Computing, laboratory of Computer Science, Systems and Optimization (ISO Lab), Ibn Tofaïl University, Kénitra, Morocco. Email: nisrine.machrafi@gmail.com

Jalal LAASSIRI, Department of Computing, laboratory of Computer Science, Systems and Optimization (ISO Lab), Ibn Tofail University, Kénitra, Morocco. Email: laassiri@uit.ac.ma to the modern internet in 1969; and put it into service. In the 80 s and by commercial providers ARPANET has been opened up to the public. In 1982, to know if sodas in a Coca-Cola's vending machine is cold, a group of programmers from Carnegie Mellon University has connected this machine to the internet. 1990: John Romkey, could turn on and off a toaster by connecting it to the Internet.

In 1993 and thanks to engineers from the University of Cambridge, the world's first webcam was created, by developing a system that takes pictures of a coffee machine three times a minute just to respect the tradition of combining the Internet with food and appliances. 1995: The birth of the first long-running GPS satellite program.

1998: IPv6 enabled more devices to interface to the internet than previously allowed by IPv4. And by that IPv6 becomes a draft standard. 1999: This is an important period for IoT, as said by Kevin Asthon, the head of MIT's Auto-ID labs in his presentation to Proctor \& Gamble executives speaking about the potential of RFID tracking technology.

2000: The internet of Refrigerators: What LG declared and has become one of the representatives of IoT engines. The idea behind it was to help all of us keep tracking of what our belonging in our fridge using screens and trackers. 2004: The birth of the phrase "Internet of Things". 2007: The first device called iPhone is here, a new gadget with a whole new figure and setting to attract and interact with the world and other Internet-connected engines. 2008: For the first time in Zurich, Switzerland, and it was the first time in the world the international IoT conference was held. 2008 was also the year when the number of connected objects outnumbered the humans in the world. 2009: Self-driving cars are being tested by Google and pacemakers (as an internet connected engines) have been released by the St. Jude Medical Center. 2010: The IoT was named as a key technology by the Chinese government and declared as a part of their development plan in a long-term.

2011: The IoT has been added as a part of the "hype cycle," the graph employed to measure the usefulness of a technology versus its popularity, by Market firm Gartner. 2013: The realization of Google Glass.

2014: The smart home hub market is helped by the release of The Echo by Amazon.

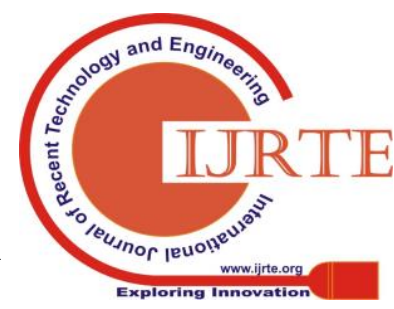


2017-2019: IoT is more democratized, since its development is more broadly-accepted, easier and cheaper, which leads to innovations in practically all industries. The IoT platforms include block chain and AI, self-driving cars are improved, and finally, smartphone/broadband penetration makes IoT a very attractive and promising option in the future.[1]

\section{B. Existing application and framework}

Until now, internets of object had touch and interfere into a lot of domain, and have several applications. Deployed extensively in:

Wearable one of the first industries field that deployed services with IoT, and these days we can see it everywhere like smart watches, heart rate monitoring, Guardian glucose monitoring device. Smart home application, this field achieved in the last decades a great popularity why its system increases quality and comfort in the daily life, using smart phones and controllers to control the smart home system. Amazon Echo is an example of smart home device application, it takes control of the smart home with owner voice, Philips Hue-Smart home lighting, the air quality Egg are also revolutionary concept of smart home devices.

Health care, IoT application can get proactive wellness-based systems by transforming reactive medical-based systems. Currently the medical research is using resources that miss critical information from the real world. It generally works with controlled environments, leftover data and volunteers for medical-examination. The focus of the internet of object now is for creation of system more than the creation of just a device; but without forgetting to increase devices power, making it more available and high precision. One of IoT advantages is the large quantity of valuable, precious data that can be analyzed and tested in real time.[1].Philips medication dispensing service is one of those devices.

Industrial automation, one of the most profound fields of the IoT and the faster development, factory digitalization, safety and security, product flow monitoring, inventory management, logistics and supply chain optimization are important as point and objectives of industrial automation.

This survey objective is presenting different architecture and protocols and gives a comparison between the different frameworks, there architectures.

This paper will provide in section 2; we briefly review the related work. In section 3 describe different architecture of IoT. Applications of connected object are presented in section 4. Next, protocols and frameworks are described in section 5. Finally, Area of application: healthcare and smart home are presented in more details in section 6 .

\section{RELATED WORK}

During this studies we get to work on different work of different researchers, some talked and discussed visions, technologies, and Areas of application like [2] and [3] which discuss the future vision, architectures, challenges and Services, and this survey explain the difference between Things oriented vision, internet oriented vision and semantic oriented vision, give an overview in challenges and present the different application of the internet o things.

We are interested in this work to know the different architecture we can work with in the IOT, so more than one article and survey gives us the more global answer we can get:

On one side [4] a study on architectures, protocols and application of IoT, present the Three, five, six layer architecture and the difference between both, and also definition of every layer on both models .

In another side [5] it's an overview of IoT presenting architecture, protocols and challenges and defining five architecture models: Three-layer Architecture which is the most general model, Middle-wave Architecture, SOA Architecture, five layer architecture which is the development of the Three layer one, and finally the cloud-specific architecture.

In the other hand this paper [6] resume the idea that architecture used in a connected object depend on the domain of application, and relay each area (agriculture , healthcare, industry, smart cities and other). In [7] Social Internet of Things: Applications, architectures and protocols.

\section{A. Challenges in the IoT}

While going further, internet of thing face several challenges which searchers and developers have to face and to find a solution.[8] Is presenting heterogeneity and communication or routing and networking challenges, and there are the general one, their also some unique challenges like wireless sensor network.

Working on this works and others, we also learn about protocol, frameworks, and application. And we will provide in the next sections more details about each one.

\section{DIFFERENT ARCHITECTURES OF IOT}

\section{A. high level architecture}

Good quality plagiarism software/ tool (Turnitin / iThenticate) will be used to check similarity that would not be more than $20 \%$ including reference section. In the case of exclusion of references, it should be less than $5 \%$.

This is the basic model of architecture with three layers, but they are other that are more sufficient for research on IoT. And it contains the perception and the network layer and the applications one, and this is the role of each one:

- Deliberation of specific services of the application to the user is the application layer responsibility. By its role internet of things can be used in multiple smart applications like health, homes and cities.

- Connection to other smart things, network devices, and servers it's a network layer responsibility. The transmitting and the processing sensor data can also be executed by the feature of this layer.

- The perception layer which is actually the physical layer, to sense and gather data from and about the current environment its use sensors. These sensors sense the physical setting and parameter, it can also spot other smart objects in the same environment.[4] 


\section{B. Middle-wave architecture}

It is basically a software or collection of sub-layers amidst technological and application layer. It addresses services such as reliability, scalability, coordination, etc., it is responsible for connecting various parts of network and gives a link for transmitting information among various LANs [5]

\section{Architecture on five layers}

Replace the network layer by two layers the transport and the processing one, and added a layer which is the business layer, and hi sis the role of each one:

- The transport layer ensures the transfer of the sensor data between the perception layer and the processing layer in both ways through networks such as wireless, 3G, LAN, Bluetooth, RFID, and NFC.

- The middleware layer which is the processing one. This layer handles a large quantity of data coming from the transport layer by storing it, analyzing it, and treating it. It can manage and provide a diverse set of services to the lower layers. It employs many technologies such as databases, cloud computing, and big data processing modules.

- The business layer is monitoring the global IoT system, the IoT system involve business and profit models, applications and users privacy. [4]

Also in defence for example there are the Four layer architecture which is Physical Sensing Layer, Gateway Communication Layer, C4ISR Management Layer, and Application Layer[5], also the SOA architecture has 4-layers communicating with each other : application layer, service and network layer and finally the perception one.[6]

\section{Fog architecture}

There is also a fog architecture with six layers which inserts four layers between the physical layer and the transport one: monitoring, pre-processing, storage, and security layers. :

- Power, services, resources and responses are controlling by Monitoring layer.

- Filtering, processing, and analytics of sensor data are performed by the pre-processing layer.

- The temporary storage layer provides storage functionalities such as data replication, distribution, and storage.

- Encryption, decryption and ensures data integrity and privacy are performed by the security layer. And before sending data to the cloud the monitoring and pre-processing are execute on the edge of the network.

This architecture was established to overcome security issues, to enforce the security attacks and prevent IoT application from it.

The connection across which data moves in any IoT architecture can be in one of this mode: Device to device, gateway to data system, device to gateway or between data systems, and the Data system could be a data center or cloud center.

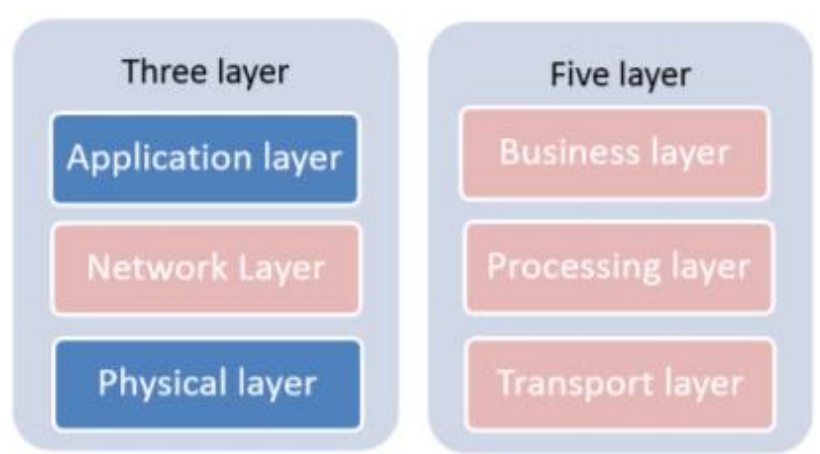

Figure 1: Three layer with five layer architecture

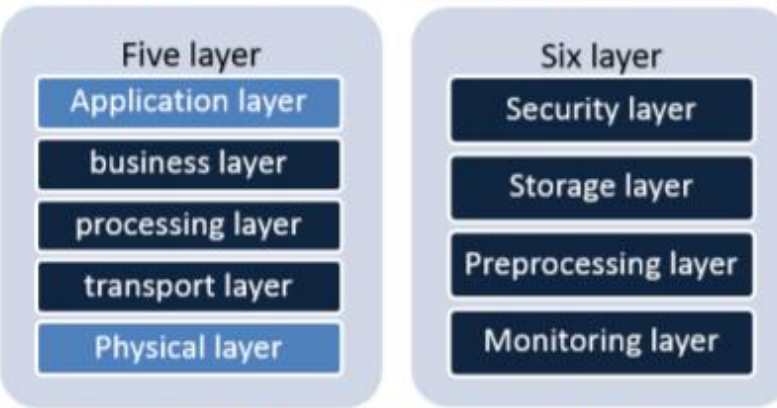

Figure 2: Five layer with six layer architecture

\section{THE DIFFERENT PROTOCOLS USED IN IOT}

The IoT protocols are classified to for Application and messaging or networks and transport or Physical devices and Communication or for management and authentication keys, each of his intervention domains has a responsible layer(s) depending on tasks.

\section{A. Application and Messaging}

The layers responsible from application and messaging are using specific protocol for the aims of transferring data or messages.

The MQ Telemetry Transport one of the common standards protocols called mostly MQTT which is maintained by OASIS, this protocol is designed for IoT communications over TCP. MQTT 3.1.1 and MQTT 5.0. MQTT the two current versions used, was developed to meet gateway messaging needs to device, and doesn't meet most requirements of gateway to an intra-datacenter or datacenter connections.

Each protocol performs in a layer or more depending of the adopted architecture, the MQTT protocol work on the top of transfer layer in the application one; using the TCP/IP to transfer messages between both level of the architecture. It was created by Doctor Andy Standford-Clarck and Arlen Nipper to suite for constrained engines resources. It is open, weightless, clear and simple to use and deploy protocol, which makes it good choice for communication in environments with constraints resources like IoT [9]. The design aim of MQTT protocol is to ensure safe delivery of message in constrained environments such as low unreliable networks and network bandwidth for resource constrained devices. And the message delivery is done by client-server publish-subscribe messaging protocol [9].

Sun Microsystems has created the standard JSM, which is an asynchronous messaging technology, and one of the most efficient and powerful one on the market;

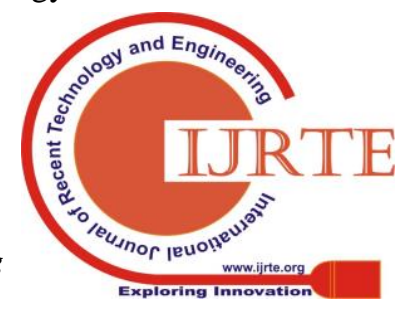


it builds the messaging systems by defining an API. Even it is not a protocol of messaging, it can build on top of different messaging protocols; which mean it define a standard of APIs without going throw specification of a protocol[10]. It extensively used already to integrate databases, and business process automation devices with a message bus and analytics devices. JMS is mainly applied in Java applications. Which mean the choice can be made among the different existing providers of JSM, but the interaction of JMS stacks on the network can't be done all the time. JMS is convenient within data-center and for gateway to the data-center connections. Today the API defined by JSM is far the most integrated on process engines, analysis engines or monitoring platforms, this standard has moved away from being the mail stack today or the most consumed by today's companies.

The AMQP (Advanced Message Queuing Protocol) is a wire-level binary protocol that allows communication between any AMQP client with any other AMQP server, with any programming platforms or languages. AMQP is the only protocol workable for end-to-end use in some specific use cases, even if it's not adapted for sensor devices with limited power; memory or network bandwidth. As an example, the device and its network with a sustainable capacity of the factory machines or the SCADA system in the Industrial IoT[11].

The RESTfull interactions depend on HTTP functions, which mean that the client side don't require any client library. Which may be useful for with simple device or sensor since only one-ways communication is needed, because it signifies that any service receiving Restful Posts can receive data from that device or sensor. The compromise with this protocol that you don't get any behavior of a messaging protocol. Unless the device or sensor application handles buffering and retries in the application code, all the data are lost ones the server become backlogged or unavailable. There are of course benefits to a messaging protocol in an end-to-end system like an IoT application.

The Constrained Application Protocol (CoAP) is based on REST methods and other ones which is a subset of HTTP, but works only with UDP not TCP, adding a service with limited quality. It was designed for constrained device connectivity in the early days of IoT's emergence - it stands for "Constrained Application Protocol." Since MQTT's arrival as a standard, with its equal handling of constrained devices, and much broader feature set beyond that, few people are choosing CoAP for new efforts. You are likely to choose CoAP only if it is the embedded choice for a sensor device, and you must support an application that already uses it [12].

To connect device to device with the lowest overhead the DDS was designed, DDS acronym for Data Distribution Service. A direct device to device data bus is implementations of DDS. To work with DDS, you create a set of topics, with their own data types. Instead of relying on a message broker, data publishers and consumers get matched through the data bus based on their types, topics and quality of service (QoS) parameters. Also it can run over UDP (multicast), TCP, shared memory, and other proprietary networks. Instead of relying on the transport layer for reliability, it has its own per-stream reliability protocol.

\section{B. Network and Transport}

A key IP (Internet Protocol)-based technology is 6LowPAN (IPv6 Low-power wireless Personal Area Network). Rather than being an IoT application protocols technology like Bluetooth or ZigBee, 6LowPAN is a network protocol that defines encapsulation and header compression mechanisms. The standard has the freedom of frequency band and physical layer and can also be used across multiple communications platforms, including Ethernet, Wi-Fi, 802.15.4 and sub-1GHz ISM[13].

IPv6(no IoT without it ), Aeron , RPL, RO(Routing protocol for low-power and lossy networks), based on: RFC6282 standard, its frequency is (adapted and used over a variety of other networking media including Bluetooth Smart (2.4GHz) or ZigBee or low-power RF (sub-1GHz).

\section{Physical devices and communication}

ZigBee protocol, used in smarts home mostly; is a wireless networking protocol basically openly available global standard to address the unique needs of low-power, low-cost wireless M2M (machine-to-machine) networks and also IoT. It operates on IEEE 802.15.4 physical radio specification and operates even in unlicensed band including $2.4 \mathrm{GHz}$, $900 \mathrm{MHz}$ and $868 \mathrm{MHz}$.

This protocol provides low-cost, low power solution for remotely controlling and monitoring smart equipment, fleet applications, home automation systems, medical devices and other compliant systems. It is used by leading manufacturers of semiconductor devices, technology firms, and wireless communication devices as the de-facto communication protocol. ZigBee 3.0 is based on IEEE802.15.4 its Frequencies $2.4 \mathrm{GHz}$, and has a distance range of [10-100]m and a data rates range $250 \mathrm{kbps}$.

The LoRaWAN protocol's is a MAC (media access control) protocol for wide area networks. Its design allows low-powered gadget to communicate with Internet-connected programs over long range wireless connections. LoRaWAN is a second and third-layer mapped protocol in the OSI model. The implementation is on top of FSK or LoRa modulation in ISM (industrial, scientific and medical ) radio bands[14].Low power wide area networks (LoRaWAN) had a diverse frequency, its distance range: $2-5 \mathrm{~km}$ in an urban environment, in suburban environment it is $15 \mathrm{~km}$ and its data rates range, [0.3-50] kbps.

\section{Physical devices and communication}

The protocol OAuth 2.0 is the industry-standard protocol for authorization. OAuth 2.0 supersedes the work done on the original OAuth protocol created in 2006. OAuth 2.0 focuses on client developer simplicity while providing specific authorization flows for web applications, desktop applications, mobile phones, and living room devices. This specification and its extensions are being developed within the IETF OAuth Working Group[15].

The OpenID is a protocol related to the OAuth 2.0 protocol, so that the first serves as an identify layer on the top of the second.

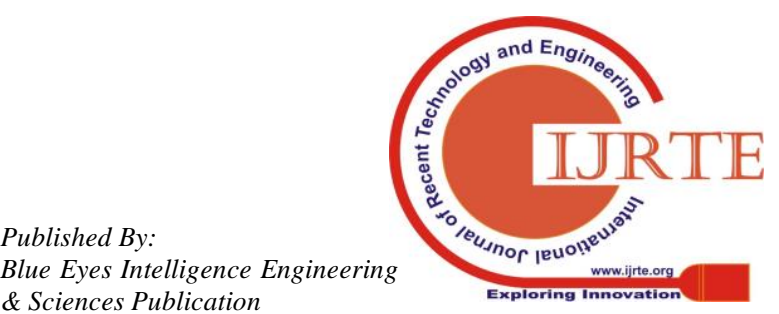


Through this protocol the end-user verification is done by the clients, this verification and the obtaining of the basic profile information's is done on the basis of authentication established by an authorization server in an interoperable and REST-like manner. [16]. to machine environment for network sensors, that's why it is a management protocol, its design is made to remotely administrate devices and enable the association of services. The foundation of this modern architectural design is REST, and an extensible resource and replying on a secure data transfer standard named the Controlled Application Protocol and that's the CoAP protocol. Moreover, with LwM2M has responded to the huge market need with OMA SpecWorks for the management of light and low-power devices on the variety of networks that can support the potential of IoT. A group of industry experts at the OMA SpecWorks Device Management Working Group has specified LwM2M based on the IETF security standards. Also, OMA DM built by the Open Mobile Alliance, but more oriented to mobile applications. Ideal for things in movement (changing IP address for instance). More complex and structured tan LwM2M [17].

The TR-069 is created by the Broadband Form (its first version is from 2004) and used in hundreds of thousands of devices all over the world, being a protocol widely used by telecommunication operators to provision their routers, etc. For this, is very complex, heavy and structured, but it works very well for gateways and telecommunications devices [17].

\section{DIFFERENT FRAMEWORKS OF INTERNET OF THINGS}

Frameworks are the result of a programming platform, which is a combination of devices, systems, architectures, applications, web services, hardware and software communicating with each other in models to get finally a framework

The AWS (Amazon Web Services) a framework using MQTT protocol and it is an administrated cloud platform that lets connected devices interact with cloud applications and other devices easily and securely. AWS IoT had a high capacity of memory it may support billions of devices and trillions of messages, and can process and route those messages to AWS endpoints and to other devices safely and securely. With AWS IoT, your applications can keep track of and communicate with all your gadget, all the time, even when they aren't connected, AWS IoT makes it easy to use AWS services like Amazon Kinesis, Amazon S3, Amazon DynamoDB, Amazon CloudWatch, and AWS CloudTrail, to build IoT applications that gather, process, analyse and act on data generated by connected devices, without having to manage any infrastructure.[18]

ARM mbed IoT is a platform to develop applications for the IoT based on ARM micro-controllers. The mbed device interface support a lot of communication protocols as ZigBee, Wi-Fi, Bluetooth, 6LoWPAN and a many other. Azure IoT Suite also a platform composed of set of services released by Microsoft, and its natively supports communication over AMQPs, MQTT or http protocols [19].
LWM2M is designed in response to the needs for machine

Eclipse has an IoT project which is offering a platform to manage control interfaces between the local of the physical IoT and the public internet or cellular network and it's called Kura.

Google had released a platform too, Brillo/Weave, it is composed of two main backbones Brillo (its operating is android-based system and weave (a communication shell message passing and for interactions). Apple had released a framework for the IoT too and it is HomeKit and it is supported only by home connected IoT equipment. The device side support ZigBee and Z-Wave.

Released by Ericsson, is an open source platform Calvin to manage and build distributed applications. The development process of this framework is divided into four steps : describe where Data is processed on the input ports of actors and when passed to output ports in order to fire some actions depending on the contents of messages/tokens. [20] , after that connect using calvinScript used to describe application and how actors connected inside them, it get the application ready and identified to be deployed. Final steps of the process are deployment and management.

\section{AREA OF IOT APPLICATION}

\section{A. Healthcare application of IOT}

For Hospitalized patients with the need for constant attention required by their physiological state the non-invasive monitoring system is the ideal solution, IoT-driven. This system uses sensors to gather physiological information, and this information will be analysed and stored employing gateways and the cloud. This data is then delivered by wireless to the stuff in charge of medical care for further analysis and consequence review, avoiding a constant verification of vital signs of patients, in a regular interval and by a health professional. Instead, it gives an uninterrupted automated flow of data. In this way, the quality of care is improved by constant care and attention which reduce the cost of care and eliminates the need for a presence of a caregiver to actively participate in data collection and analysis [21].

In Order to get Data, analysing it and respond to it, IoT use MQTT, HTTP or CoAP protocols, ZigBee can be used too.

HTTP, an application level protocol for distributed, collaborative, hypermedia information systems, can be used as a name servers or distributed object management systems, this protocol is not just for hypertext uses. CoAP used to equip constrained environments with HTTP web transfer mechanisms. The key difference between HTTP and CoAP is related to underlying transport layer. HTTP relies on connection-oriented TCP protocol, while CoAP is built on top of simple connections UDP protocol [22].

MQTT send data over low bandwidth networks and define quality of service QoS,

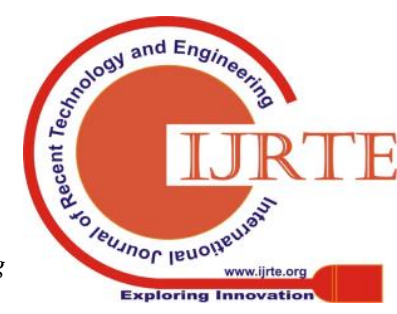


The connection operation enables MQTT as an optimal connection protocol for IoT and M2M.ZigBee is widely used in connected object as smart home, industry and healthcare, other protocols can be used and was used but ZigBee can overcome issues faced by other protocols, as power consumption, scalability.

Different farm had developed architecture adapted to their services and connected objects they product, Figure 3 proposed AWS-based architecture for real-time remote health monitoring of patients.

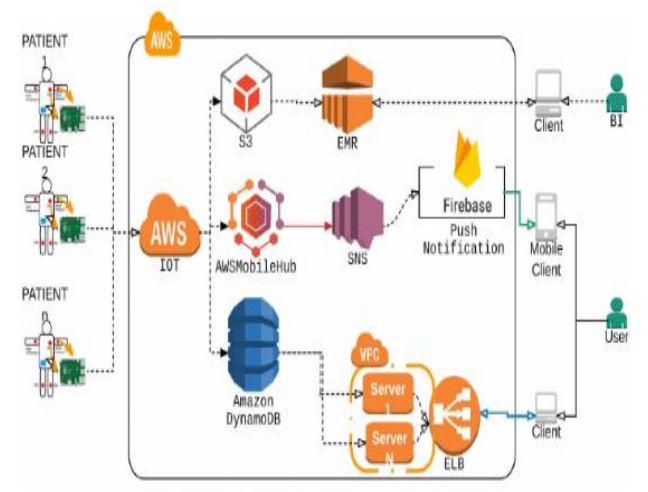

\section{Figure 3: Architecture of the proposed health monitoring systems}

To reach this system, sensors are installed on patients to collect medical data and transmit it using MQTT protocol to the AWS IoT gateway for processing and storage[23].

Some example for application of Internet of things in Healthcare field:

\section{i. Monitoring an aging relative}

The Technology based on ultrasound is already used and deploy in hospitals can be used as a solution to locate and find an aging resident in a home with specific healthcare setting it can be used also to detect incident. A System with battery-powered (on economic mode) is set up for emergency calls via an easy-to-install interface with an extensive communication.

A system that performs well for senior home monitoring can be a small waterproof sensor system, worn like a wristwatch. At regular intervals of 15 to 20 seconds, the system is programmed to send a positioning signal to the ultrasound receiver. This receiver, on receiving the signal communicates over standard wireless WLAN connections to the homecare gateway. Continuous analysis of the data is done by the gateway. Relevant data is broadcast, and the built-in wireless wide area network connection is used to send out a notification for help as soon as any critical event is detected.

\section{ii. Scalable, continuous, heart rate monitoring}

Each patient has his own anthropology which must be monitored and controlled independently, so each patient's biometrics will have parameters having its threshold belonging to a specific patient. This means that the heart rate of an ECG can be monitored, the level of activity too, the heart rate and even the position of the body can be monitored. Other devices can be added to this system for remote monitoring of blood pressure, weight and other vital signals. This is not all in the health field or patient monitoring, there are clinical applications to understand the symptoms after the medical therapy for arrhythmias in order to monitor the effectiveness of the treatment taken by the patient, the post ablation procedure to track vital signs and heart rate defined by monitoring breathing and cardiac activity remotely whether in the hospital or at home.

\section{B. Smart home}

An intelligent environment takes the role of an intelligent agent controlling and monitoring by evaluating the state of a residence and the physical environment for its occupants through sensors, and even proceed on this environment through effectors to optimize the specified performance measures. [24]. The performance measures to be maximized in a smart home can be comfort and productivity of its inhabitants, whereas those to be minimized are the operation costs (i.e. cost of energy and other utilities)[25].

Now, some cities, like Barcelona, are investing on technologies and development of IoT services for mobility and traffic management, and there are some industrial products, like DYNC ATMS (Advanced Traffic Management System) [26] , Siemens COMET [27] or TransSuite [28], provide highway monitoring, traffic light management functionalities.

\section{CONCLUSION}

As the sections of this paper make it clear, IoT word can't be stopped any more, every day a new device is created and developed, to ameliorate privacy, security, efficiency more protocols and more frameworks and on the top of that more architectures see a new day.

The timeline of IoT become richer each year, the biggest farm invests more to the developer the field of intervention with internet of thing, being more creative on the hardware and the software making daily life more comfortable.

The mean idea is that with every creative step of the Internet of Things, to get to the other step, result with be shown for that simulator of IoT join the game: netSIM, Iotify and other will be present in future works.

\section{REFERENCES}

[1] Upasana, "Real World IoT Applications in Different Domains," 2019 [Online]. Available: https://www.edureka.co/blog/iot-applications/. [Accessed: 19-Oct-2019].

[2] A. Rghioui and A. Oumnad, "Internet of Things: Visions, Technologies, and Areas of Application," vol. 5, no. 6, pp. 83-91, 2017.

[3] D. Singh, "A survey of Internet-of-Things: Future Vision , Architecture , Challenges and Services," no. March, 2014.

[4] P. Sethi and S. R. Sarangi, "Internet of Things: Architectures Protocols , and Applications," vol. 2017, 2017.

[5] P. Aswale, A. Shukla, P. Bharati, S. Bharambe, and S. Palve, An Overview of Internet of Things: Architecture, Protocols and Challenges. Springer Singapore.

[6] P. P. Ray, "A survey on Internet of Things architectures," J. King Saud Univ. - Comput. Inf. Sci., vol. 30, no. 3, pp. 291-319, 2018. 
[7] S. Rho and Y. Chen, "Social Internet of Things: Applications , architectures and protocols," Futur. Gener. Comput. Syst., vol. 92, pp. 959-960, 2019

[8] Z. H., H. A., and M. M., "Internet of Things (IoT): Definitions, Challenges and Recent Research Directions," Int. J. Comput. Appl., vol. 128, no. 1, pp. 37-47, 2015.

[9] S. Manandhar, "Srijan Manandhar Mqtt Based Communication in Iot," no. May, 2017.

[10] G. Bouloukakis, "Enabling emergent mobile systems in the IoT: from middleware-layer communication interoperability to associated QoS analysis Georgios Bouloukakis To cite this version: HAL Id : tel-01592623 e Pierre et Marie Curie emes Mobiles Emergents dans 1' IoT :," 2018.

[11] Solace, "Understanding IoT Protocols - Matching your Requirements to the Right Option | Solace." [Online]. Available: https://solace.com/blog/understanding-iot-protocols-matching-requir ements-right-option/. [Accessed: 13-Jul-2019].

[12] Solace, "Understanding IoT Protocols - Matching your Requirements to the Right Option | Solace," 2017. [Online]. Available: https://solace.com/blog/understanding-iot-protocols-matching-requir ements-right-option/. [Accessed: 07-Sep-2019].

[13] RS Components, "11 Internet of Things (IoT) Protocols You Need to Know About," 2015. [Online]. Available: https://www.rs-online.com/designspark/eleven-internet-of-things-iotprotocols-you-need-to-know-about. [Accessed: 07-Sep-2019].

[14] "LoRaWAN | The Things Network." [Online]. Available: https://www.thethingsnetwork.org/docs/lorawan/. [Accessed: 08-Aug-2019].

[15] "OAuth 2.0 - OAuth." [Online]. Available: https://oauth.net/2/. [Accessed: 07-Sep-2019].

[16] "OpenID Connect | OpenID." [Online]. Available: https://openid.net/connect/. [Accessed: 07-Sep-2019].

[17] "IoT device management protocols: LwM2M, OMA-DM and TR-069 Muutech Monitoring Solutions," 2019. [Online]. Available: https://www.muutech.com/en/iot-device-management-protocols-lwm 2m-oma-dm-and-tr-069/. [Accessed: 07-Sep-2019].

[18] O. S. Web, “AWS IoT Platform - Amazon Web Services.” [Online]. Available: https://www.amazonaws.cn/en/iot-platform/. [Accessed: 06-Jul-2019].

[19] “Azure IoT protocol gateway | Microsoft Docs," 2017. [Online]. Available:

https://docs.microsoft.com/en-us/azure/iot-hub/iot-hub-protocol-gate way. [Accessed: 07-Sep-2019].

[20] M. Ammar, G. Russello, and B. Crispo, "Internet of Things : A survey on the security of IoT frameworks," J. Inf. Secur. Appl., vol. 38, pp. 8-27, 2018.

[21] A. Kulkarni and S. Sathe, "Healthcare applications of the Internet of Things : A Review," vol. 5, no. 5, pp. 6229-6232, 2014

[22] D. Ugrenovic, G. Gardasevic, and IEEE, "CoAP protocol for Web-based monitoring in Io healthcare applications," Telecommun. forum, vol. 7, pp. 79-82, 2015.

[23] A. Bouslama and Y. Laaziz, "AWS For Healthcare System," no. March, 2018.

[24] W. Keith Edwards and R. E. Grinter, "At home with ubiquitous computing: Seven challenges," Lect. Notes Comput. Sci. (including Subser. Lect. Notes Artif. Intell. Lect. Notes Bioinformatics), vol. 2201, pp. 256-272, 2001.

[25] S. K. Das, D. J. Cook, A. Bhattacharya, E. O. Heierman, and T. Y. Lin, "The role of prediction algorithms in the MavHome smart home architecture," IEEE Wirel. Commun., vol. 9, no. 6, pp. 77-84, 2002.

[26] Kapsch, "Kapsch TrafficCom North America Intelligent Transportation Systems." [Online]. Available: https://www.roadtraffic-technology.com/contractors/traffic_man/tran sdyn/. [Accessed: 20-Oct-2019].

[27] "COMET ( UTMAC traffic management and information system." [Online].

Available: https://www.transportxtra.com/publications/local-transport-today/sup plements/159/13640/comet-utmc-traffic-management-and-informatio n-system-/.

[28] "Integrated Software Solutions." [Online]. Available: https://www.transcore.com/its/transsuite.

\section{AUTHORS PROFILE}

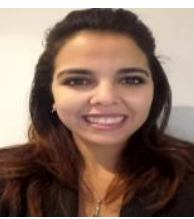

Nisrine MACHRAFI member of Informatics, System Optimization Laboratory Ibn Tofail University Kenitra, Morocco. Phd student Working on the Contributions to the Security of embedded and mobile systems. Her research interests include internet of things, security of IoT, healthcare and smart home IoT devices. Affiliated to Informatics systems and optimization Laboratory at Ibn Tofail University, Kenitra, Morocco

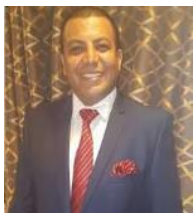

Dr. Jalal LAASSIRI member of Informatics, System Optimization Laboratory at Ibn Tofail University, Kénitra, Morocco. Laboratory Team Head he received his Ph.D. degree in computer sciences and engineering from the University of Mohammed V, Rabat, Morocco. He is a Member of the International Association of Engineers (IAENG), Dr. Laassiri authored/co-authored many Journal articles, Conference Proceedings and Book Chapters. His research interests include Wireless Sensor Networks, Network Security, Business Intelligent, Big Data, Smart-Cities, Internet of Things, He joined the Faculty of Sciences of Kénitra, Department of Computer Science, Ibn Tofail University, Morocco, as a Professor in October 2010 